\title{
Reflective Crack Mitigation Using AST Interlayer over Soil-Cement Base for Flexible Pavements
}

\author{
Mohammad Reza-Ul-Karim Bhuyan \\ mrb4081@louisiana.edu \\ University of Louisiana at Lafayette, Lafayette, Louisiana, USA \\ Mohammad Jamal Khattak \\ khattak@louisiana.edu \\ University of Louisiana at Lafayette, Lafayette, Louisiana, USA
}

\begin{abstract}
Flexible pavement over soil-cement base deteriorates over time due to reflective cracking caused by the shrinkage of such base. The propagation of the reflective cracks in overlaying hot mix asphalt (HMA) could be mitigated by installing interlayer system. State of Louisiana, USA has been utilizing Asphaltic Surface Treatment (AST) as an interlayer between the soil-cement base and HMA to mitigate reflective cracking. To evaluate the field performance of AST interlayer, flexible pavement projects with and without AST interlayer over soil-cement bases were compared in this study. From the Pavement Management System (PMS) database of Louisiana, 27 AST interlayer projects (70 miles) and 46 No AST interlayer projects (175 miles) were selected for comparison. The service lives of these projects were assessed using the time series cracking, rutting, and international roughness index (IRI) data. It was found that the projects with AST interlayer produced on average 14.3 and 14.7 years of service lives for transverse and alligator cracking, respectively. On the other hand, the average service lives of No AST interlayer projects were 11.6 and 12.5 years for such cracks, respectively. Hence, the AST interlayer projects gained 2.7 and 2.2 years of service life for transverse and alligator cracking, respectively. However, the service life based on rut data showed a loss of 3.2 years for AST interlayer projects (16.2 years). Furthermore, the longitudinal cracking and IRI exhibited no improvements. In summary, AST interlayer can mitigate transverse and alligator cracking but slightly increase the rutting potential of the pavement.
\end{abstract}

Keywords: Soil-cement base; AST interlayer; Reflective crack

\section{INTRODUCTION}

Cement stabilized soils are abundantly used in Louisiana as the base of flexible pavements due to its ease of construction, inexpensive nature and load carrying potential. Along with Louisiana, many other US states (TX, CA, MS, VA, NM, GA) are also constructing cement stabilized soil bases for last couple of decades. It's one of the available options for base construction when natural soils are not hard enough to carry extensive traffic load. But as a consequence of cement stabilization, such bases shrink during its long curing and hardening process. Hence, cement stabilized soil-cement bases always develop shrinkage cracks over time. After the construction of flexible pavement with such base, these shrinkage cracks start reflecting from the base to the overlying Hot Mix Asphalt (HMA) surface. For this reason, the cracking potential of such HMA layer 
increases substantially and it causes considerable reduction of service lives for such pavements. To increase the service lives of such pavements, many US highway agencies are now striving to control these reflective cracks. (Titi et al., 2003); (Mursalin et al., 2001); (Quintus et al., 2009); Boudreau et al., 2016); (Adaska et al., 2004); (Louw \& Jones, 2004); (Metcuff et al., 2001); (Sebesta \& Scullion, 2004) and (Wu et al., 2018).

\subsection{Reflective Crack Mitigation Techniques}

There are many different alternatives for reflective crack mitigation in the existing literature. These alternatives include but are not limited to: Micro-cracking, inverted pavement or stone interlayers stress absorbing interlayers.

\subsubsection{Micro-cracking}

At the initial curing stage (1 to 3 days) of soil-cement base construction, a network of thin hairline cracks is purposely created by the compaction of high frequency steel vibratory roller. Afterwards, the soil-cement base loses its compressive strength temporarily, but it gains that back within few months. These network of hairline cracks are called micro-cracks which avert the soil-cement base from further crack widening. Hence, the micro-cracked base minimizes or prevents crack reflection from the base. This technique is now currently on the evaluation process in Louisiana, Texas and California. (Louw \& Jones, 2004); (Sebesta \& Scullion, 2004) and (Wu et al., 2018).

\subsubsection{Inverted Pavements or Stone Interlayers}

When a pavement is constructed by installing unbound compacted aggregates (4 to 8 inches thick) between the HMA layer and soil-cement base, it is called as an inverted pavement as its cross section looks like an inverted anvil. These aggregates or stones act as an interlayer and delays crack reflection and its propagation. This technique was first augmented in South Africa back in 1970s. Here in this case, unbound compacted aggregate traps the underneath shrinkage crack as it loses the propagation path. Currently, many US states (LA, NC, VA, NM, GA etc.) are evaluating the prospective of stone interlayer as a reflective crack control method for soil-cement bases for flexible pavements. (Boudreau et al., 2016); (Buchanan, 2010); (Vaughan, 2015); (Papadopoulos, 2014).

\subsubsection{Stress Absorbing Interlayers}

Installing a stress absorbing interlayer between soil-cement base and overlying HMA layer is another technique to mitigate reflective cracking. The shrinkage cracks are temporarily halted by the interlayer membrane and its propagation is delayed for substantial time. In this case, the interlayer member provides a firm base for the overlaying layer by arresting the crack underneath. Paving Fabrics are one example of such interlayers for PCC (Portland Cement Concrete) or granular bases for flexible pavements. (Fyfe, 2010); (Nonwoven, 1997) and (Gibb, 1992). Asphalt Rubber Membrane Interlayer (ARMI) is another example of effective stress absorbing interlayer for PCC or granular bases (West, 1994 \& Scofield, 1989). Along with ARMI, several other fiber reinforced stress absorbing membrane interlayer (SAM or SAMI) are being used in USA as an effective interlayer for both PCC and HMA pavements (Metcuff et al., 2001); (Chowdhury \& Button, 2007). There is not enough literature for stress absorbing 
interlayer utilization for exactly soil-cement bases.

\subsection{AST interlayer as a reflective crack mitigation technique in Louisiana}

To mitigate or prevent reflective crack propagation on traditional soil-cement pavements, Louisiana have been implementing several techniques since 1990s. One of such techniques is the installation of AST (Asphaltic Surface Treatment) interlayer between cement stabilized soil-cement base and HMA layer. It's worth mentioning here that Asphaltic Surface Treatment (AST) is the application of a layer of liquid asphalt over the top of the soil-cement base followed by one stone thick loose aggregate spread and its compaction. If the process is repeated over the first layer of AST, then it is double layer or 2-course AST. AST is also called as Chip Seal treatment as it is a type of seal by aggregate chips. AST is usually being applied over HMA surface as a preventive type of treatment for long time but its utilization as an interlayer is relatively new and it was not found in the literature. (Nonwoven, 1997); (Gibb, 1992); (Gransberg \& James, 2005); (Johnson, 2000); (Bolander, 2005); (Hicks, 2000).

Louisiana started utilizing AST as an interlayer on soil-cement base for flexible pavements from year 2000 as a means of reflective crack mitigation. In this research study, field performance of all those AST interlayer projects (2-course) were evaluated. About 70 miles of AST interlayer projects over soil-cement base were compared with about 175 miles of No AST interlayer projects for cracking, rutting and roughness. From all these comparisons, the extension of service lives was determined which measures the effectiveness of AST interlayer.

\section{OBJECTIVES OF THE STUDY}

The main objective of this study is to evaluate the actual field performance of AST interlayer and No AST interlayer projects on soil-cement bases for all distresses (cracking, rutting and roughness). This will be accomplished through the determination of service life extension caused by the application of AST interlayer on the soil-cement bases. Thus, this study will compare the service lives of AST interlayer and No AST interlayer projects over soil-cement bases for the above distress types.

\section{RESEARCH METHODOLOGY}

To achieve the objectives of the study, an orderly research methodology was developed and that is presented below:

\subsection{Data Source and Data mining}

Office of the pavement management system (PMS) manages Louisiana DOTD's mainframe database that contains the time-series distress data of all flexible pavements. Since 1995, these distress data have been recorded. These distress data include but not limited to following distresses and index: Transverse Cracking (TC), Longitudinal Cracking (LC), Alligator Cracking (AC), Rutting and International Roughness Index (IRI). For each 1/10th mile pavement sections, all these distress data and indexes are recorded. It's worth mentioning here that a soil-cement project could be miles longer and each miles of project would include 10 pavement sections, hence 10 data points for 
all distresses. As an example, a 3.5-mile-long project would have thirty-five 1/10th mile sections or data points. For each 1/10th mile sections: TC, LC, AC, Rutting, IRI data are available from year 1995 to 2017 in PMS database. These data could be used for the performance evaluation of those $1 / 10$ th mile sections or entire projects.

With the help of the office of PMS, district engineers and information available in Treatment of Projects (TOPS), all the candidate AST interlayer and No AST interlayer projects were identified. Moreover, Traffic data and pavement system data (thickness of HMA and bases) were tabulated for each project.

\subsection{Pavement Section Selection}

With the help of MATLAB (2016b) and "MS Excel Visual Basics for Application (VBA) 2016", each 1/10th mile sections distress data (TC, LC, AC, Rutting, IRI data) were acquired from the identified candidate projects. All these time series distress data are modelled as a function of time using generic models shown in table 1. Now, as these data did not always behave properly, rational criteria were set for acceptable data selection. Hence, following criteria were augmented for the acceptation of 1/10th mile pavement section for use in these analyses:

1. Minimum three data points. Each $1 / 10$ pavement section must have at least 3 data points as any non-linear model (shown in table 1) could not be fit without 3 data points. Hence, any section that does not have at least 3 data points, were rejected for analyses.

2. Positive gain in distress. After any pavement construction, the distress values should be generally increasing over time. Hence, when the appropriate models(shown in Table 1) were fit to the distress data, the parameters $\beta, \omega, \theta$, or $\mu$ should be positive for each $1 / 10$ th mile pavement sections. If the parameters were negative, it means that the pavement is healing over time, which is not reasonable. It may happen because of small treatments which were given to the pavement section that reduces/eliminates the distress data value. Hence, those sections with negative parameters were not included in these analyses. Figure 1 shows an example of accepted and rejected section for the acceptable criteria illustration.

Table 1: Generic Pavement Performance Models (Baladi, 2015)

\begin{tabular}{|c|c|c|c|}
\hline \multirow[t]{2}{*}{ Form of equation } & \multicolumn{3}{|c|}{ Pavement distress type (model form) } \\
\hline & IRI (exponential) & Rut depth (power) & Cracking (Logistic (S-shaped)) \\
\hline $\begin{array}{l}\text { Generic equation } \\
\text { (modelling) }\end{array}$ & $I R I=\alpha \exp t \beta$ & $R u t=\gamma t^{\omega}$ & $\operatorname{Crack}=\frac{\operatorname{Max}}{1+\exp }$ \\
\hline $\begin{array}{l}\text { Service Life }(\mathrm{SL})= \\
\text { Time to reach thresh- } \\
\text { old }\end{array}$ & $t=\frac{\ln \left(\frac{\text { Threshold }}{\alpha}\right)}{\beta}$ & $t=\exp \left[\frac{\ln \left(\frac{\text { Threshold }}{\gamma}\right)}{\omega}\right.$ & $t=\left[\frac{1}{\alpha}\left(\ln \left(\frac{\text { Max }}{\text { Threshold }}\right)-1\right)\right]-\left(\frac{\beta}{\alpha}\right)$ \\
\hline
\end{tabular}



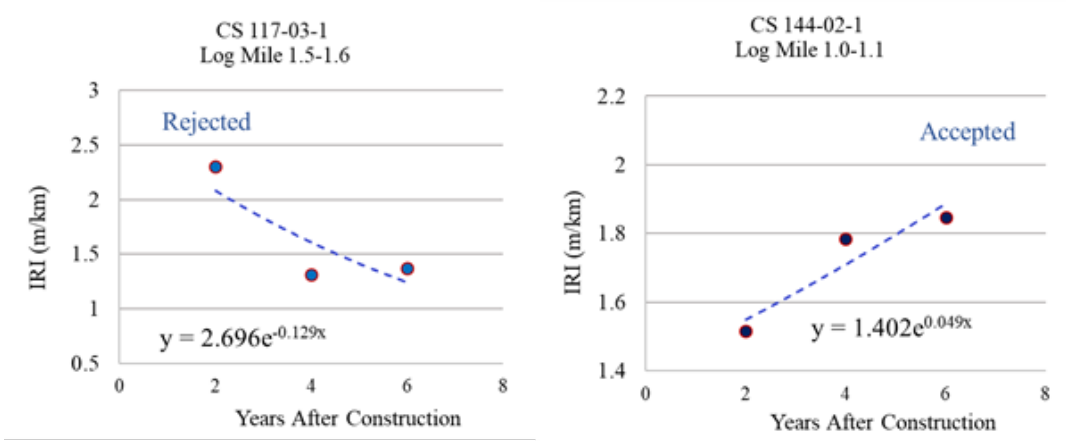

Figure 1: Acceptance Criteria Illustration

Now, any pavement sections have almost zero cracking/rutting and minimum roughness immediately after construction. Hence, one initial data point could be reasonably assumed for a better fit and it also increases the number of accepted data points. Therefore, initial values for distress data were assumed as $0.079 \mathrm{~m}, 0.67 \mathrm{~m} / \mathrm{km}$ and $0.0254 \mathrm{~cm}$ for cracking, rutting and IRI, respectively. Also, small values $(0.3048 \mathrm{~m})$ for cracking are assumed when crack is zero at any year after construction as non-linear model cannot take zero values.

\subsection{Service Life Computations}

To evaluate the performance of AST interlayer pavements, the service lives of 1/10th mile sections are calculated from table 1 for each distress types (TC, LC, AC, Rutting, IRI). The threshold values of all distress types for SL computations were selected as 3.2 $\mathrm{m} / \mathrm{km}, 1.27 \mathrm{~cm}, 322.5 \mathrm{~m}, 322.5 \mathrm{~m}$ and $117.7 \mathrm{~m} 2$ for IRI, Rut, Transverse Cracking (TC), Longitudinal Cracking (LC), Alligator Cracking (AC), respectively. MATLAB (2016b) and Excel VBA (2016) was utilized to gather and organize all the pavement sections. Using the models shown in table 1, the service lives of all sections were calculated. Its worth mentioning here that any pavement section that generates more than 20 years of service lives by the models, are considered to have only 20 years service life, as the design life of these pavements were only 20 years. Also, all the sections shown here have ESAL: 0-30000, thickness of HMA: 0-4 inches and they have 5 to 7 years of surface age. All AST and No AST soil-cement pavement sections were gathered separately and a histogram of service lives were generated for each of the two categories for comparison. Figure 2 shows the histogram comparison of Transverse Cracking for AST and No AST pavement sections: 


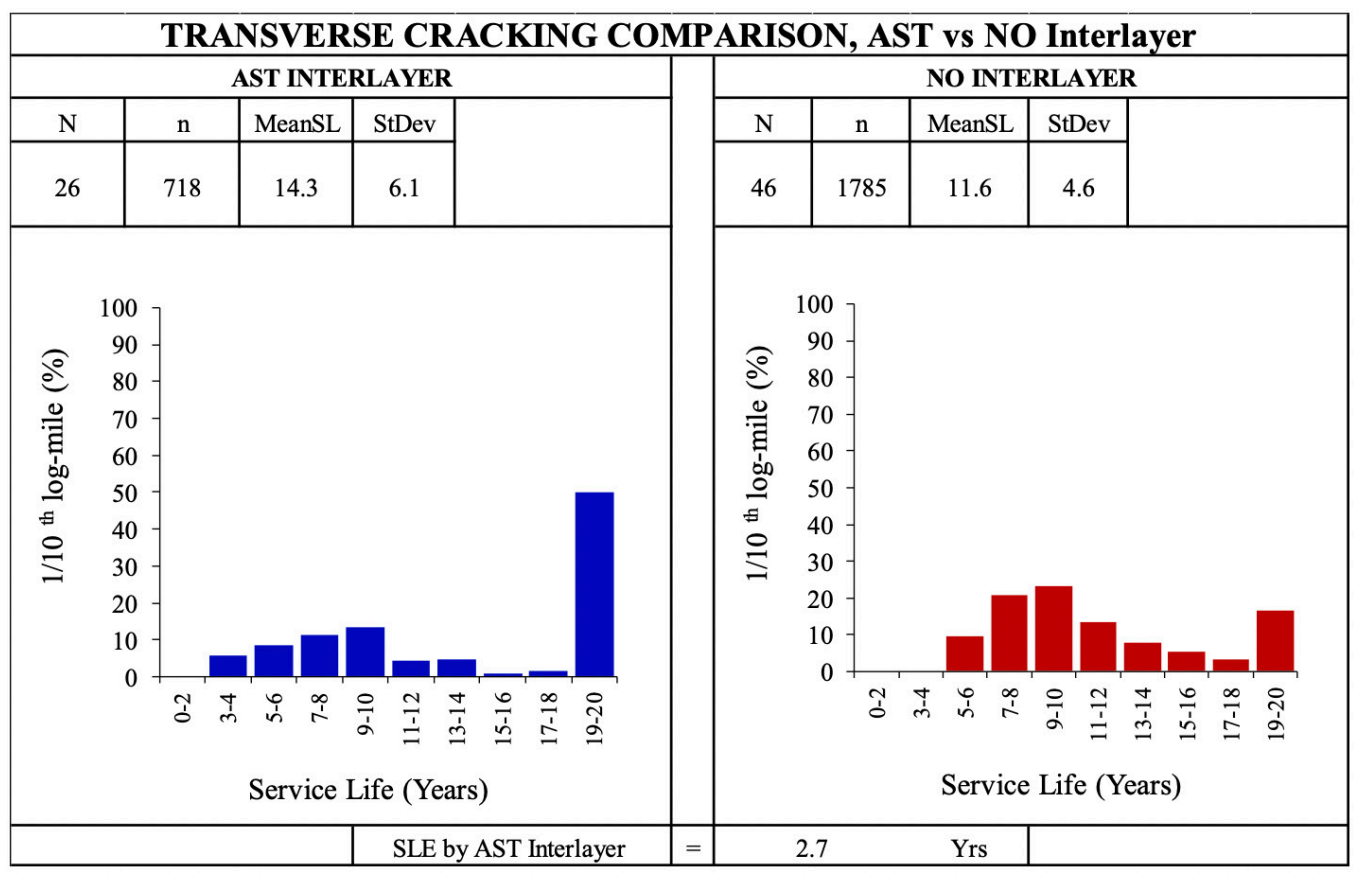

Figure 2: Transverse Cracking Comparison, AST vs No AST interlayer

Percentage of sections are shown at the y-axis of the histogram and service lives (with a bin of 2 years interval) plotted at the $\mathrm{x}$-axis. The arithmetic mean of all service lives of all sections (MeanSL, as shown in the figure 2) were calculated for both AST and No AST sections. Standard Deviation (StDev, as shown in the figure 2) of service lives were also shown in this figure. Here in this figure: $\mathrm{N}=$ number of projects available for the group, $\mathrm{n}=$ number of accepted 1/10th miles sections for the group. Now, from Figure 2, it is obvious that 26 AST interlayer projects were accepted which includes 718 $1 / 10$ th miles of sections ( $=71.8$ miles). The arithmetic mean of the services lives of these sections were 14.3 years and these sections have a standard deviation of 6.1 years. For No AST interlayer, 46 projects with 1785 1/10th miles (=178.5 miles) of sections were accepted for analysis and these sections have a MeanSL of 11.6 years.

Finally, Service Life Extension (SLE) is calculated to evaluate the performance of AST interlayer by the following equation:

$$
\text { SLE }=\text { MeanSLAST }- \text { MeanSLNoAST }
$$

Here, the Service Life Extension (SLE) for TC is 2.7 years (14.3-11.6=2.7 years) for AST interlayer. It means, on average, AST pavements provide 2.7 years of service life extension when compared to No AST pavements. From the histogram comparison, it is clear that AST pavements are better than No AST pavements as far as TC is concern. Here, $50 \%$ of AST section has 20 years of SL whereas only $20 \%$ of No AST section has 20 years of service life.

Similar figures were developed for AC and Rutting and shown in the next chapter. SLE is calculated for each of the distress types and the prospective of AST as interlayer 
were evaluated from those SLE values. It's worth mentioning here that SLE could be either of positive or negative values. If SLE values are negative, it would mean that AST had reduced the service lives of pavement on average.

\section{RESULTS AND DISCUSSION}

Figure 3 and Figure 4 show the histogram comparison of $\mathrm{AC}$ and Rutting. From figure 3 , it is manifest that AST interlayer extends the life of pavements by 2.2 years on average. Moreover, $60 \%$ of sections for AST interlayer had minimum 20 years of service life. Whereas, only $30 \%$ sections had minimum 20 years of service life for No AST interlayer sections. There are enough data present on both sides in this comparison, hence these results are conclusive.

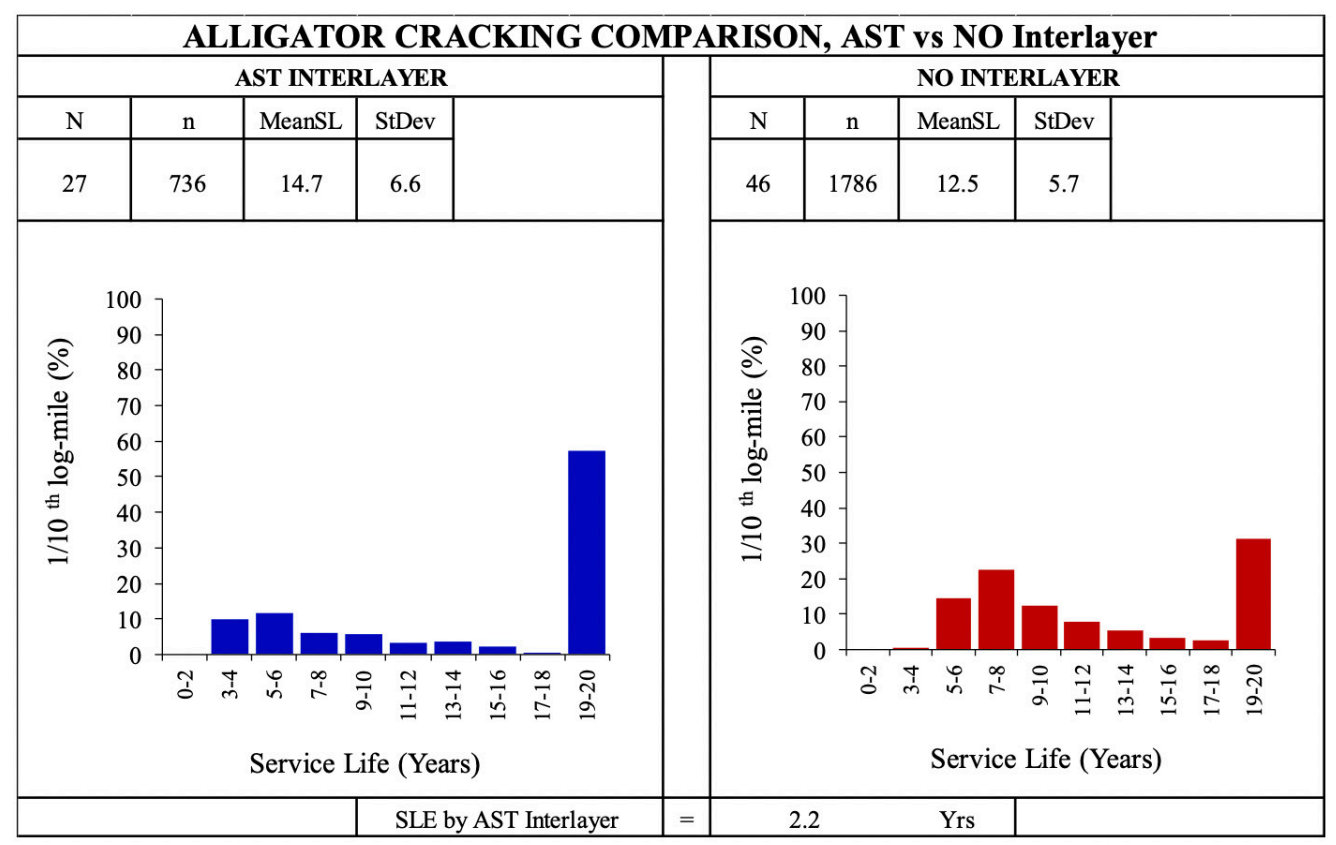

Figure 3: Alligator Cracking Comparison, AST vs No AST interlayer

The rutting potential of AST and No AST interlayer sections were evaluated at figure 4. The AST interlayer sections here behaved worse than the No AST interlayer sections. There is no failure of pavements for No AST sections, as almost $100 \%$ sections had minimum 20 years of service lives (MeanSL $=20.0$ years). But for AST interlayer sections, only around $60 \%$ sections had minimum 20 years of service lives, another $40 \%$ (around) sections failed before the pavements design service life of 20 years. These $40 \%$ sections service lives are distributed between 5 to 19 years, which could be ascertained from the histogram. As a result, the MeanSL for rutting reduces significantly from 20.0 years to 16.8 years. Consequently, the SLE for rutting became negative: -3.2 years. 


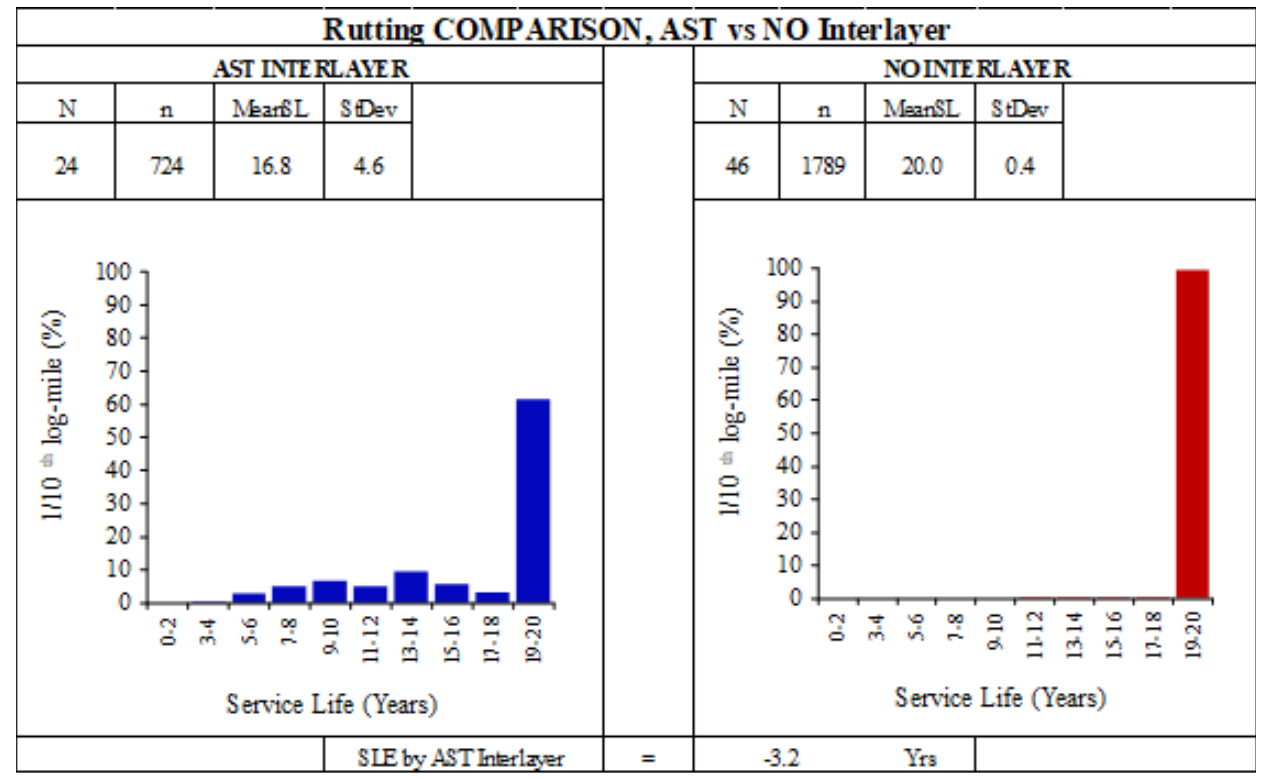

Figure 4: Rutting Comparison, AST vs No AST interlayer

Similar analyses were performed for LC and IRI and it was found that MeanSL for No AST and AST pavements were 16.0 and 15.3 years, respectively for LC; 19.7 and 18.3 years, respectively for IRI. Subsequently, the SLE by AST interlayer is -0.6 years and -1.3 years, respectively for LC and IRI. Hence, roughness and longitudinal crackings are largely unaffected by AST interlayer, as their MeanSL were very similar and SLE values were not large enough.

To summarize, AST interlayer had improved cracking performance of flexible pavements over soil-cement bases, as it extends the service lives of pavement sections by 2 to 3 years (for TC and $\mathrm{AC}$ ). It also does not change the overall roughness and LC potential of pavement sections. But AST interlayer creates unwanted rutting to the pavement and reduces the service lives for around 3 years for rutting.

\section{CONCLUSION}

Here in this research, the performance of 70 miles of AST interlayer projects were compared with 175 miles No AST interlayer projects over soil-cement bases. Based on the comparisons, it was found that AST interlayer did mitigate reflective cracking by extending the average service lives of pavements by 2 to 3 years for Transverse and Alligator cracking. In case of longitudinal cracking and IRI, the performance of pavements was largely unaffected by AST interlayer. As far as rutting is concerned, AST interlayer exhibits undesirable rutting in the pavements, and it reduces the service lives by 3 years due to rutting. As these results are supported by more than 70 miles of distress data, the findings of this study are conclusive. Hence, AST interlayer is only recommended as a reflective crack mitigation technique when the rutting of pavements is not a concern for any highway agency. 


\section{ACKNOWLEDGEMENTS}

The authors would like to thank Mark Martinez, Kevin Gaspard as well as project review committee for their efforts and valuable input to assist in the data acquisition and project identifications. Special thanks to LTRC, DOTD to fund the research project and University of Louisiana to accommodate the research.

\section{REFERENCES}

Adaska, W.S. \& D. Luhr. (2004). Control of Reflective Cracking in Cement Stabilized Pavements. In 5th International RILEM Conference, France, 2004.

Baladi, G. \& Khattak, M. J. (2015). Development of Cost Effective Treatment Performance and Treatment Selection Models. Louisiana Department of Transportation and Development.

Boudreau, R., Vaughan, K. \& Frost, D. (2016). Interved Pavements. [TRB Webinar]. Washington, D.C.

Bolander, P. (2005). Seal Coat options: taking out the mystery. In First National Conference on Pavement Preservation, Missouri.

Buchanan, S. (2010) Inverted Pavement Systems, South Africa.

Chowdhury, A. \& Button, J. (2007) Evaluation of FiberMat ${ }^{\circledR}$ Type B as a Stress Absorbing Membrane, Texas Transportation Institute, Texas A\&M University, College Station, Texas.

Fyfe, R. (2010) Geotextile Reinforced Seals Under Asphalt. Department of Transport, Energy and Infrastructure, South Australia, New South Wales.

Gibb, I. (1992). Chip Sealing in the Shire of Broome Australia. In Geosynthetics World, London, the UK.

Gransberg, D., James, D. M. B. (2005). Chip Seal Best Practices. NCHRP Synthesis of Highway Practice, 342.

Hicks, G.R., Seeds, S.B. \& Peshkin, D.G. (2000) Selecting a preventive maintenance treatment on flexible pavements. Foundation of Pavement Preservation.

Johnson, A. (2000). Best practices Handbook on Asphalt Pavement Maintenance. Center of Transportation Studies.

Louw, S. \& Jones, D. (2015). Pavement Recycling: Literature Review on Shrinkage Crack Mitigation in Cement-Stabilized Pavement Layers. University of California: Pavement Research Center, Technical Memorandum.

Metcuff, J. B., Roberts, F. L. \& Rasoulin, M. (2001) Construction and Comparison of Louisiana's Conventional and Alternate Base Courses Under Accelerated Loading. Louisiana Transportation Research Center, Baton Rouge.

Mursalin, M., Titi, H., Martinez, M., Bercel, B. \& Keel, G. (2001) Long Term Performance of Stone Interlayer Pavement. Louisiana Transportation Research Center.

Nonwoven Paving Fabrics Study, Industrial Fabrics Association International, Austin, TX, 1997.

Papadopoulos, E. G. (2014). Performance of Unbound Aggregate Bases And Implications For Inverted Base Pavements. Georgia Institute of Technology.

Quintus, H. L., Mallela, V. J., Weiss, W. \& Shen, S. (2009). Techniques for Mitigation of Reflective Cracks. Applied Research Associates, Inc.

Scofield L. A. (1989). The History, Development and Performance of Asphalt Rubber at ADOT. Arizona Transportation Research Center, Phoenix, Arizona. 
Sebesta, S. \& Scullion, T. (2004) Effectiveness Of Minimizing Reflective Cracking In Cement Treated Bases By Microcracking. Texas Transportation Institute.

Titi, H., Rasoulian, M., Martinez, M., Becnel, B. \& Keel, G. (2003). Long-Term Performance of Stone Interlayer Pavement. Journal of Transportation Engineering, ASCE.

Vaughan, K. (2015). Inverted Pavement. Vulcan Materials Company, South Africa.

West, K. (1994). Field Performance of Asphalt-Rubber Interlayers. Texas Transportation Institute, Austin, Texas.

Wu, Z., Liu, Y. \& Intaj, F. (2018) Minimizing Shrinkage Cracking in Cement-Stabilized Bases through Micro-Cracking. Louisiana Department of Transportation and Development, Baton Rouge. 